\title{
Vulnerability Analysis of Electric Power Communication Network
}

\author{
Yucong $\mathrm{Wu}$ \\ Department of Telecommunications Engineering, School of Electrical and Electronic Engineering, \\ North China Electric Power University, Beijing, 102206, China
}

346591653@163.com

\begin{abstract}
Keywords: Electric Power Communication Network; Vulnerability; Measure of the Fragility; Characteristics Index Evaluation Method; Business Relative Important Matrix
\end{abstract}

\begin{abstract}
As a support network of the smart grid, the reliability of electric power communication network has become the prerequisites of the smart grid to become intelligent and economic, and operate safely. This paper mainly studies the vulnerability of the electric power communication network, so as to provide a strong guarantee and support for electric power production. Vulnerability, as an effective measure of network reliability, can measure the performance of the network under attack or equipment failure. Here we put forward an analysis method about the vulnerability of the electric power communication network business layer, based on the importance of electric power business: in view of the uncertainty produced during expert evaluation link in importance evaluation of traditional electric power business, we put forward an objective standards instead of experts subjective rating business importance evaluation method, namely characteristic index evaluation method.
\end{abstract}

\section{Introduction}

With the booming of electric power technology, electric system higher the requirements on communication security. The vulnerabilities in electric power communication system lead to incidents. In the traditional analysis, we use reliability index to analyze the electric power communication system. The reliability index only relies on the amount of known incident type, which is unable to reflect the defect of the system and it is not conducive to improve the system. However, the evaluation based on the concept of vulnerability synthesize the safety index, reflect the system security level in general. The network vulnerability, as an effective measure to the system reliability, reflects decline degree of network performance on different network unit and point out the weak link in the network, which supports network planning and risk management.

In this paper, we first state the concept of electric power telecommunication network and its main business. Second, we elaborate the development status of electric power communication network. Then we discuss the basic concept of the vulnerability and its characteristics. After that we confirm the measurement index of the vulnerability.

At last, we put forward an electric power communication network business layer model of its vulnerability. In this model, an electric power business importance evaluation method is proposed, in which the expert subjective rating is replaced by objective indicators. It eliminates the subjective uncertainty of evaluation results. Then we use business importance as parameters. We build the electric power communication network model on the business layer and analyze the vulnerability of electric power communication network in various link failure model. At last, we adjust the weak link in the network according to the results to improve the network performance. 


\section{The concept of electric power communication network}

Power Line Communication, whose full name is Power Line Carrier Communication, is a special communication method using high voltage power lines, medium voltage power line and low voltage power line as information transmission medium for voice or data transmission.

Network vulnerability as an effective measure of network reliability measures the network performance when network is attacked or equipment naturally fails. It reflects various decline degree when different network unit fails and point out the weak link in the network to support the network programming and risk management.

Electric power communication business is divided to production business and management business. There are a large amount of indexes to describe network vulnerability, such as integrity, expansivity and so on. In the traditional network vulnerability analysis, we consider rather less on categories and characteristics of network transmission business, which has its limitation.

\section{Development of electric power communication network}

Public network is lack of communication capacity and develops slowly. There are various types of business in the public communication network. The business with huge span makes it difficult to refine the network performance index to the business layer. As the electric communication network special for electric system, the demand of improving communication capability and satisfy to meet the special request of the electric system arises.

Because of the specificity of the electric system, power generation, transmission, substation, power distribution is conducted at the same time and distribution area is very extensive. In order to reasonable economic power generation, distribution of electricity and timely find and deal with the faults of power system, we have to set up an electric power system for unified management and need a safeguard of electric power communication system to supervise the whole process of electric power system and detection.

These days, electric power communication network in China has made great progress with the development of the power system. After years of development, communication institutions and team should have a certain scale and to create a good academic atmosphere. Even so, there are still some defects in our country contrast to the big trend in the development of the global telecommunications and direction of the development of electric power communication around the world electric power communication network at present.

The network structure is weak. The main electric power communication network structure is complex, mainly tree or star structure, difficult to form a roundabout circuit. Hence, it is difficult to reasonable share through the detour once the fault happens.

Communication system is relatively backward and main circuit over term service. Many circuits operate for ages, over the term of scrap. It is difficult to complete the integrated digital business.

Network access and network management is weak. The current user access is telephone lines in general or the analog signal interface. Difficult as the network management information is collected, it is hard to form a comprehensive network management system.

\section{The basic concept of vulnerability}

In system safety point of view, vulnerability refers to exist in a weakness or defect in the system, system for a specific threat attack or the sensitivity of risk events and the possibility of the threat of attack effect. The index of vulnerability and other indexes have fundamental difference. 
Generally, system security measures have effectiveness, reliability calculation and anti-destroying ability calculation.

The reliability refers to the system's ability to fully realize some function in a certain time. Validity refers to the system is enabled at any time in efficient working condition. Anti-destroying ability is in view of the network topology structure reliability assessment. Anti-destroying ability of the network node communication is mainly pointing to the safety degree of the system under destruction.

Thus, the fragility of the description angle and the form are different to the above two cases. It focus on vulnerable part of the network after attack, brought about by the decline in overall performance.

\section{Measure of the fragility}

Nowadays, there is no unified definition of Fragility. Fragility which we used in the paper is the performance decline of some nodes in the network after attack. Currently, the basic parameters of measuring the network in the complex network theory characteristics are the shortest path, clustering coefficient, degree, degree distribution and so on. These parameters show the characteristics of the network performance from different aspects.

Average distance L. In a network, the distance between any two nodes is defined as the shortest distance $d_{i, j}$ between the two notes. We get the average distance from the node distance. It reflects the average length of information transmission between nodes in the network,

$$
L=\frac{1}{n(n-1)} \sum_{i \neq j} d_{i j}
$$

Clustering coefficient $\mathbf{C}$. The clustering coefficient in the network is the important parameters used to evaluate network node cluster. The quantity of clustering coefficient shows the degree of collectivization in the network,

$$
C=\frac{1}{n} \sum_{i=1}^{n} C_{i}
$$

Node degree distribution $P_{c u m}(k)$. Degree distribution effects greatly on the network transmission performance of the information. We use $\mathrm{P}\left(\mathrm{k}^{\prime}\right)$ to indicate the percentage of number of nodes with $k^{\prime}$ degree and the accumulate probability of notes degree is

$$
P_{\text {cum }}(k)=\sum_{k^{\prime} \geq k} P\left(\mathrm{k}^{\prime}\right)
$$

Node betweenness distribution $P_{c u m}(S)$. In the network, node betweenness is the frequency if passing the note in the shortest distance. We use $\mathrm{P}\left(\mathrm{S}^{\prime}\right)$ to indicate the percentage of number of nodes with $S^{\prime}$ betweenness and the accumulate probability of notes betweenness is

$$
P_{\text {cum }}(\mathrm{S})=\sum_{\mathrm{S}^{\prime} \geq S} P\left(\mathrm{~S}^{\prime}\right)
$$

Generally we use these parameters to indicate network structure characteristics. However it is unable to describe the network performance comprehensively. Currently, the index we use to evaluate the network performance is efficiency function. The efficiency index of the network $G$ is defined as 


$$
E(G)=\frac{1}{n(n-1)} \sum_{i, j \in G, i \neq j} \varepsilon_{i j}=\frac{1}{n(n-1)} \sum_{i, j \in G, i \neq j} \frac{1}{d_{i j}}
$$

We use $\varepsilon_{i j}$ to indicate the communication efficiency between two notes and it is $\varepsilon_{i j}=\frac{1}{d_{i j}}$, inversely with the shortest distance between the two notes. When $d_{i j}=\infty$, that is, when there are no path between the two notes, $\varepsilon_{i j}=0$.

The function of the communication system is different from the electric system. Hence the analytic angle is not the same. In this paper, we consider the communication network business impact from two aspects: the business transmission bandwidth and connectivity. The original efficiency function only indicates the business transmission bandwidth and it doesn't involve the connectivity. Hence we will modify the efficiency function. The result is as follows:

The method of calculating the shortest path doesn't change. Then we calculate the total efficiency $\mathrm{E}$ of the system.

We get the total business volume $\mathrm{S}$ from the business matrix $S_{i j}$ and calculate the shortest distance matrix $A_{i j}$. Of those, $a_{i j}$ is the shortest distance between note i and $\mathrm{j}$.

We wipe off one dots every time we do the simulation and count the business which loses link. Then we represent the efficiency function by

$$
E^{\prime}=\frac{\sum_{i \neq j, a_{i j} \neq 0} S_{i j}}{\sum_{i \neq j} S_{i j}} E
$$

In the equation, $\mathrm{E}$ is the efficiency function calculated by the original method.

$\sum_{i \neq j, j, a_{i j} \pm S_{i j}} S_{i j} E$

is the percentage of the transporting business, which is regarded as business connected efficiency. The efficiency after modified not only considers the business connectivity, but also the effect of business distribution to network transmission characteristics. When the note with more business connection fails, the value of $\sum_{i \neq j}^{\sum_{i \neq, i, a_{0}} S_{i j}} E$ and $\mathrm{E}$ is small.

\section{Characteristics index evaluation method}

Business relative important matrix. Expert evaluation in the traditional electric power business importance evaluation has strong subjectivity. Different experts grading results vary, which may lead to inconsistent evaluation and affect all sorts of network performance analysis based on the business important degree. In order to eliminate the effect, we need to find objective factors replacement for the expert subjective factors to describe the important degree of each other between the business.

Different electric power business have different requirements on the same technical indicators. Some indicators cannot directly reflect the importance of business, such as communication bandwidth. However, some index reflects the important degree of business characteristics, such as time delay. We define the index that can reflect the business important degree as characteristic index, and use it as an objective evaluation factors instead of the expert subjective factors. Then we evaluate electric power business importance through the analysis of the different requirements of business on the characteristic indexes.

We select the collection of characteristic indexes $K=\left\{k_{n}\right\}, n=1,2, \ldots, N$ and for business collection $B=\left\{b_{i}\right\}, i=1,2, \ldots, I$ map various requirements of the indexes to integer field forming important value sequence $\left\{s_{i}\left(k_{n}\right)\right\}, s_{i}\left(k_{n}\right) \in\left\{1,2, \ldots, S_{k_{n}}\right\} s_{i}\left(k_{n}\right)$ represents the importance value of business $b_{i}$ under the characteristic value $k_{n}$. For the business requires highest on characteristic 
$k_{n}, s_{i}\left(k_{n}\right)=S_{k_{n}}$. For the business requires lowest on characteristic $k_{n}, s_{i}\left(k_{n}\right)=1$. $S_{k_{n}}$ is determined by difference degree of business set under the feature index $k_{n}$, of which the value has $S_{k_{n}}$ different requirements to index $k_{n}$. We get the business relative important matrix $A^{\left(k_{n}\right)}$ under the characteristic index $k_{n}$ through the calculation of business important value sequence,

$$
A^{k_{n}}=\left[\begin{array}{cccc}
a_{11}^{k_{n}} & a_{12}^{k_{n}} & \cdots & a_{1 I}^{k_{n}} \\
a_{21}^{k_{n}} & a_{22}^{k_{n}} & \cdots & a_{2 I}^{k_{n}} \\
& \ddots & \\
a_{I 1}^{k_{n}} & a_{I 2}^{k_{n}} & \cdots & \\
a_{I I}^{k_{n}}
\end{array}\right]
$$

In the equation, $a_{i j}^{\left(k_{n}\right)}$ is whether the business $b_{j}$ important under characteristic index $k_{n}$ relative to the business. $1=$ important, $0=$ unimportant, $0.5=$ of the same importance as $b_{j}$.

When $i=j, a_{i i}^{\left(k_{n}\right)}$ has no practical meaning and it should be a value that doesn't affect the result. According to the algorithm, we have $a_{i i}^{\left(k_{n}\right)}=0$ in this paper. When $i \neq j$,

$$
a_{i j}{ }^{\left(k_{n}\right)}=\left\{\begin{array}{c}
1, \frac{s_{i}\left(k_{n}\right)}{s_{j}\left(k_{n}\right)}>1 \\
0.5, \frac{s_{i}\left(k_{n}\right)}{s_{j}\left(k_{n}\right)}=1 \\
0, \frac{s_{i}\left(k_{n}\right)}{s_{j}\left(k_{n}\right)}<1
\end{array}\right\}
$$

We sum up all business relative important matrix under all indexes in the collection of characteristic index and get synthetically relative important matrix $\boldsymbol{A}$, in which,

$$
a_{i j}=\sum_{n=1}^{N} a_{i j}{ }^{\left(k_{n}\right)}
$$

The evaluation of business importance. In order to avoid the difference between business important degree evaluation result is too large or too small. In this paper we use the linear normalization function and interval mapping function to deal with synthetically relative important matrix and get important business degrees. First of all, we sum up the row vector elements of the synthetically relative important matrix and get the sum of all the other synthetically relative important value of focusing business $b_{i}$ to business,

$$
a_{i}^{\text {sum }}=\sum_{j=1}^{I} a_{i j}
$$

Then, we select the linear normalization function

$$
f(z)=\frac{z-z_{\min }}{z_{\max }-z_{\min }}
$$

In the equation, $z$ is the variate to be normalized and $z_{\max }, z_{\min }$ respectively represents the maximum and the minimum variable in $z$ domain, $f(z) \in[0,1]$. We substitute $a_{i}^{\text {sum }}$ into equation and get normalized important value:

$$
a_{i}^{\prime}=f\left(a_{i}^{\text {sum }}\right)=\frac{a_{i}^{\text {sum }}-\left(a_{i}^{\text {sum }}\right)_{\min }}{\left(a_{i}^{\text {sum }}\right)_{\max }-\left(a_{i}^{\text {sum }}\right)_{\min }}
$$

After normalization, the minimum value of $a_{i}^{\prime}$ is 0 , which represents the importance degree of the business is 0 , namely it can be discarded, which is not reasonable. Therefore, we need to map $a_{i}^{\prime}$ through formula 


$$
Q_{i}=Q\left(a_{i}{ }^{\prime}\right)=a_{i}^{\prime}(1-X)+X
$$

to interval $[X, 1]$, in which $0<X<1$ and get the reasonable importance evaluation $Q_{i}$ of business $b_{i}$. When the value of $X$ is too large, the difference of importance degree between business is too small and it is difficult to distinguish various business. Hence, in this paper, we have $X=0.1$ to define the distance between the maximum and minimum of importance degree as ten times.

\section{Reference}

[1] Zhaoxia X, Manimaran G, Vittal V. An information architecture for future power systems and its reliability analysis[J]. IEEE Transactions on Power Systems,2002,17(3): 857-863.

[2] Barefoot C A, Entringer R, Swart H. Vulnerability in graphs-a comparative survey [J]. Journal of Combinatorial Mathematics and Combinatorial Computing 1987(1):13-22.

[3] Dinh T N, Ying Xuan, Thai M T, et al. On new approaches of assessing network vulnerability: hardness and approximation[J]. IEEE/ACM Transactions on Networking, 2012，20(2): 609-619.

[4] Vardi Y, Zhang Cun Hui. Measures of network vulnerability[J]. IEEE Signal Processing Letters, 2007, 14(5): 313-316.

[5] Peng Wei, Li Zimu, Liu Yujing, et al. Assessing the vulnerability of network topologies under large-scale regional failures[J]. IEEE Journal of Communications and Networks, 2012, 14(4): 451-460.

[6] Wang Xiaoliang, Jiang Xiaohong, Pattavina A. Assessing network vulnerability under probabilistic region failure model[C]//IEEE 12th International Conference on High Performance Switching and Routing (HPSR). Cartagena, Spain: IEEE, 2011.

[7] ZHAO L, PARK K, and LAI Y C. Attack vulnerability of scale-free networks due to cascading breakdown[J], Phys. Rev. E, 2004(70): 35-101. 